UDC 517.54

\author{
A. K. Bakhtin, I. V. Denega
}

\title{
SHARP ESTIMATES OF PRODUCTS OF INNER RADII OF NON-OVERLAPPING DOMAINS IN THE COMPLEX PLANE
}

\begin{abstract}
In the paper we study a generalization of the extremal problem of geometric theory of functions of a complex variable on non-overlapping domains with free poles: Fix any $\gamma \in \mathbb{R}^{+}$and find the maximum (and describe all extremals) of the functional

$$
\left[r\left(B_{0}, 0\right) r\left(B_{\infty}, \infty\right)\right]^{\gamma} \prod_{k=1}^{n} r\left(B_{k}, a_{k}\right),
$$

where $n \in \mathbb{N}, n \geqslant 2, a_{0}=0,\left|a_{k}\right|=1, B_{0}, B_{\infty},\left\{B_{k}\right\}_{k=1}^{n}$ is a system of mutually non-overlapping domains, $a_{k} \in B_{k} \subset \overline{\mathbb{C}}$, $k=\overline{0, n}, \infty \in B_{\infty} \subset \overline{\mathbb{C}},(r(B, a)$ is an inner radius of the domain $B \subset \overline{\mathbb{C}}$ at $a \in B)$. Instead of the classical condition that the poles are on the unit circle, we require that the system of free poles is an $n$-radial system of points normalized by some "control" functional. A partial solution of this problem is obtained.

Key words: inner radius of a domain, non-overlapping domains, radial system of points, separating transformation, quadratic differential, Green's function
\end{abstract}

\section{Mathematical Subject Classification: 30C75}

Let $\mathbb{N}, \mathbb{R}$ be the sets of natural and real numbers, respectively, $\mathbb{C}$ be the complex plane, $\overline{\mathbb{C}}=\mathbb{C} \bigcup\{\infty\}$ be a one-point compactification, and $\mathbb{R}^{+}=(0, \infty)$. Let $\chi(t)=\frac{1}{2}\left(t+t^{-1}\right), t \in \mathbb{R}^{+}$, be the Zhukovskii function. Let $r(B, a)$ be an inner radius of the domain $B \subset \overline{\mathbb{C}}$ relative to the point $a \in B$.

The system of points $A_{n}:=\left\{a_{k} \in \mathbb{C}, k=\overline{1, n}\right\}, n \in \mathbb{N}, n \geqslant 2$ is called $n$-radial, if $\left|a_{k}\right| \in \mathbb{R}^{+}$for $k=\overline{1, n}$ and $0=\arg a_{1}<\ldots<\arg a_{n}<2 \pi$.

Denote

$$
P_{k}=P_{k}\left(A_{n}\right):=\left\{w: \arg a_{k}<\arg w<\arg a_{k+1}\right\}, \quad a_{n+1}:=a_{1},
$$

(C) Petrozavodsk State University, 2019 


$$
\alpha_{k}:=\frac{1}{\pi} \arg \frac{a_{k+1}}{a_{k}}, \quad \alpha_{n+1}:=\alpha_{1}, \quad k=\overline{1, n}, \quad \sum_{k=1}^{n} \alpha_{k}=2 .
$$

For any $n$-radial system of points $A_{n}=\left\{a_{k}\right\}, k=\overline{1, n}$, we introduce the "control" functional

$$
\mathcal{L}\left(A_{n}\right):=\prod_{k=1}^{n} \chi\left(\left|\frac{a_{k}}{a_{k+1}}\right|^{\frac{1}{2 \alpha_{k}}}\right) \cdot\left|a_{k}\right| .
$$

The class of $n$-radial systems of points for which $\mathcal{L}\left(A_{n}\right)=1$ contains automatically all systems of $n$ different points of the unit circle.

Consider the following extremal problem.

Problem 1. For any fixed value of $\gamma \in \mathbb{R}^{+}$, find the maximum of the functional

$$
J_{n}(\gamma)=\left[r\left(B_{0}, 0\right) r\left(B_{\infty}, \infty\right)\right]^{\gamma} \prod_{k=1}^{n} r\left(B_{k}, a_{k}\right)
$$

where $n \in \mathbb{N}, n \geqslant 2, a_{0}=0, A_{n}=\left\{a_{k}\right\}_{k=1}^{n}$ are $n$-radial systems of points, such that $\mathcal{L}\left(A_{n}\right)=1, B_{0}, B_{\infty},\left\{B_{k}\right\}_{k=1}^{n}$ is a system of mutually non-overlapping domains, $a_{k} \in B_{k} \subset \overline{\mathbb{C}}, k=\overline{0, n}, \infty \in B_{\infty} \subset \overline{\mathbb{C}}$; also, describe all extremals.

This problem belongs to the class of extremal problems with free poles. Problems of this type have been studied in many papers (see, for example, [1-16]). For $\gamma=\frac{1}{2}$ and $n \geqslant 2$, an estimate of the functional $J_{n}(\gamma)$ for the system of non-overlapping domains was found in the paper [6, p. 59]. Kuz'mina [15, p. 267] strengthened this result for simply connected domains and showed that the estimate is correct for $\gamma \in\left(0, \frac{n^{2}}{8}\right], n \geqslant 2$. Note that for $n=2$ the Kuz'mina's estimate of the functional (1) coincides with the Dubinin's estimate. Some partial cases of the above-posed problem were considered in $[2,3,5]$.

Let

$$
\begin{aligned}
S(x) & =x^{2 x^{2}+2} \cdot|1-x|^{-(1-x)^{2}} \cdot(1+x)^{-(1+x)^{2}} \text { and } \Psi(x)=\ln (S(x)) . \\
\Psi^{\prime}(x) & =4 x \ln (x)-2(x-1) \ln |x-1|-2(x+1) \ln (x+1)+\frac{2}{x}(\text { see Fig. } 1) .
\end{aligned}
$$

The function $S(x)$ is logarithmically convex on the interval $\left[0, x_{0}\right]$, $x_{0} \approx 0.88441$. Let $\Psi^{\prime}(x)=t, y_{0} \leqslant t<0, y_{0} \approx-1.06$. The equation $\Psi^{\prime}(x)=t_{k}$ has two solutions $x_{1}(t) \in\left(0, x_{0}\right]$ and $x_{2}(t) \in\left(x_{0}, \infty\right]$. 


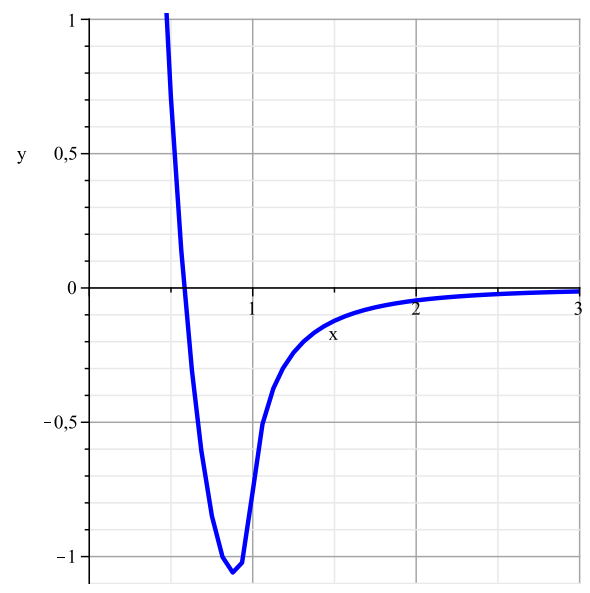

Figure 1: The function plot $y=\Psi^{\prime}(x)$

Let $\delta_{n}^{0}=\min \left((n-1) x_{1}(t)+x_{2}(t)\right)=2 \sqrt{\gamma_{n}^{0}}$, then $\gamma_{n}^{0}=\left(\frac{\delta_{n}^{0}}{2}\right)^{2}$. Then the following proposition is true.

Theorem 1. Let $n \in \mathbb{N}, n \geqslant 2, \gamma \in\left(0, \gamma_{n}^{0}\right], \gamma_{n}^{0}=\left(\frac{\delta_{n}^{0}}{2}\right)^{2}$. Then, for any $n$-radial system of points $A_{n}=\left\{a_{k}\right\}_{k=1}^{n}$ such that $\mathcal{L}\left(A_{n}\right)=1$, and any system of mutually non-overlapping domains $B_{0}, \quad B_{\infty}, B_{k}$, $a_{0}=0 \in B_{0} \subset \overline{\mathbb{C}}, \infty \in B_{\infty} \subset \overline{\mathbb{C}}, a_{k} \in B_{k} \subset \overline{\mathbb{C}}, k=\overline{1, n}$, the following inequality holds:

$$
\left[r\left(B_{0}, 0\right) r\left(B_{\infty}, \infty\right)\right]^{\gamma} \prod_{k=1}^{n} r\left(B_{k}, a_{k}\right) \leqslant\left[r\left(\Lambda_{0}, 0\right) r\left(\Lambda_{\infty}, \infty\right)\right]^{\gamma} \prod_{k=1}^{n} r\left(\Lambda_{k}, \lambda_{k}\right),
$$

where the domains $\Lambda_{0}, \Lambda_{\infty}, \Lambda_{k}$, and the points $0, \infty, \lambda_{k}, k=\overline{1, n}$, are, respectively, circular domains and poles of the quadratic differential

$$
Q(w) d w^{2}=-\frac{\gamma w^{2 n}+\left(n^{2}-2 \gamma\right) w^{n}+\gamma}{w^{2}\left(w^{n}-1\right)^{2}} d w^{2}
$$

Proof. Let $\zeta=\pi_{k}(w)$ denote a univalent branch of the multivalent analytic function $-i\left(e^{-i \arg a_{k}} w\right)^{\frac{1}{\alpha_{k}}}, k=\overline{1, n}$, that maps $P_{k}$ onto the right halfplane $\operatorname{Re} \zeta>0$ conformally in the one-sheet way. Consider the system of functions $\zeta=\pi_{k}(w)=-i\left(e^{-i \arg a_{k}} w\right)^{\frac{1}{\alpha_{k}}}, \quad k=\overline{1, n}$. Let $\Omega_{k}^{(1)}, k=\overline{1, n}$, denote a domain of the plane $\mathbb{C}_{\zeta}$, obtained as a result of the union of the 
connected component of the set $\pi_{k}\left(B_{k} \bigcap \bar{P}_{k}\right)$, containing the point $\pi_{k}\left(a_{k}\right)$, with its symmetric reflection with respect to the imaginary axis. In turn, by $\Omega_{k}^{(2)}, k=\overline{1, n}$, we denote the domain of the plane $\mathbb{C}_{\zeta}$, obtained as a result of the union of the connected component of the set $\pi_{k}\left(B_{k+1} \bigcap \bar{P}_{k}\right)$, containing the point $\pi_{k}\left(a_{k+1}\right)$, with its symmetric reflection with respect to the imaginary axis, $B_{n+1}:=B_{1}, \pi_{n}\left(a_{n+1}\right):=\pi_{n}\left(a_{1}\right)$. In addition, $\Omega_{k}^{(0)}$ denotes a domain of the plane $\mathbb{C}_{\zeta}$ obtained as a result of the union of the connected component of the set $\pi_{k}\left(B_{0} \bigcap \bar{P}_{k}\right)$, containing the point $\zeta=0$, with its symmetric reflection with respect to the imaginary axis. Similarly, $\Omega_{k}^{(\infty)}$ denotes a domain of the plane $\mathbb{C}_{\zeta}$ obtained as a result of the union of the connected component of the set $\pi_{k}\left(B_{\infty} \cap \bar{P}_{k}\right)$, containing the point $\zeta=\infty$, with its symmetric reflection with respect to the imaginary axis. It is clear that $\pi_{k}\left(a_{k}\right):=\omega_{k}^{(1)}, \pi_{k}\left(a_{k+1}\right):=\omega_{k}^{(2)}, k=\overline{1, n}, \pi_{n}\left(a_{n+1}\right):=\omega_{n}^{(2)}$. The definition of the functions $\pi_{k}$ yields

$$
\begin{gathered}
\left|\pi_{k}(w)-\omega_{k}^{(1)}\right| \sim \frac{1}{\alpha_{k}}\left|a_{k}\right|^{\frac{1}{\alpha_{k}}-1} \cdot\left|w-a_{k}\right|, \quad w \rightarrow a_{k}, \quad w \in \bar{P}_{k}, \\
\left|\pi_{k}(w)-\omega_{k}^{(2)}\right| \sim \frac{1}{\alpha_{k}}\left|a_{k+1}\right|^{\frac{1}{\alpha_{k}}-1} \cdot\left|w-a_{k+1}\right|, \quad w \rightarrow a_{k+1}, \quad w \in \bar{P}_{k}, \\
\left|\pi_{k}(w)\right| \sim|w|^{\frac{1}{\alpha_{k}}}, \quad w \rightarrow 0, \quad w \in \bar{P}_{k}, \\
\left|\pi_{k}(w)\right| \sim|w|^{\frac{1}{\alpha_{k}}}, \quad w \rightarrow \infty, \quad w \in \bar{P}_{k} .
\end{gathered}
$$

Using the corresponding results for the separating transformation $[6,7]$, we get the inequalities

$$
\begin{gathered}
r\left(B_{k}, a_{k}\right) \leqslant\left[\frac{r\left(\Omega_{k}^{(1)}, \omega_{k}^{(1)}\right) \cdot r\left(\Omega_{k-1}^{(2)}, \omega_{k-1}^{(2)}\right)}{\left.\frac{1}{\alpha_{k}}\left|a_{k}\right|^{\frac{1}{\alpha_{k}}-1} \cdot \frac{1}{\alpha_{k-1}}\left|a_{k}\right|^{\frac{1}{\alpha_{k-1}}-1}\right]^{\frac{1}{2}},}\right. \\
r\left(B_{0}, 0\right) \leqslant\left[\prod_{k=1}^{n} r^{\alpha_{k}^{2}}\left(\Omega_{k}^{(0)}, 0\right)\right]^{\frac{1}{2}}, \\
r\left(B_{\infty}, \infty\right) \leqslant\left[\prod_{k=1}^{n} r^{\alpha_{k}^{2}}\left(\Omega_{k}^{(\infty)}, \infty\right)\right]^{\frac{1}{2}} .
\end{gathered}
$$

The conditions of realization of the sign of equality in inequalities $(4)-(6)$ are described in [7, p. 29]. On the basis of those relations, we 
get the inequality

$$
\begin{gathered}
J_{n}(\gamma) \leqslant\left(\prod_{k=1}^{n} \alpha_{k}\right) \prod_{k=1}^{n} \frac{\left|a_{k}\right|^{\frac{1}{\alpha_{k}}}+\left|a_{k+1}\right|^{\frac{1}{\alpha_{k}}}}{\left(\left|a_{k}\right|\left|a_{k+1}\right|\right)^{\frac{1}{2 \alpha_{k}}}} \cdot\left|a_{k}\right| \times \\
\times\left\{\prod_{k=1}^{n}\left(r\left(\Omega_{k}^{(0)}, 0\right) r\left(\Omega_{k}^{(\infty)}, \infty\right)\right)^{\gamma \alpha_{k}^{2}} \cdot \frac{r\left(\Omega_{k}^{(1)}, \omega_{k}^{(1)}\right) \cdot r\left(\Omega_{k}^{(2)}, \omega_{k}^{(2)}\right)}{\left(\left|a_{k}\right|^{\frac{1}{\alpha_{k}}}+\left|a_{k+1}\right|^{\frac{1}{\alpha_{k}}}\right)^{2}}\right\}^{\frac{1}{2}} .
\end{gathered}
$$

Further, from the last relation we have

$$
\begin{gathered}
J_{n}(\gamma) \leqslant\left(\prod_{k=1}^{n} \alpha_{k}\right) \prod_{k=1}^{n}\left(\left|\frac{a_{k}}{a_{k+1}}\right|^{\frac{1}{2 \alpha_{k}}}+\left|\frac{a_{k+1}}{a_{k}}\right|^{\frac{1}{2 \alpha_{k}}}\right)\left|a_{k}\right| \times \\
\times\left\{\prod_{k=1}^{n}\left(r\left(\Omega_{k}^{(0)}, 0\right) r\left(\Omega_{k}^{(\infty)}, \infty\right)\right)^{\gamma \alpha_{k}^{2}} \cdot \frac{r\left(\Omega_{k}^{(1)}, \omega_{k}^{(1)}\right) \cdot r\left(\Omega_{k}^{(2)}, \omega_{k}^{(2)}\right)}{\left(\left|a_{k}\right|^{\frac{1}{\alpha_{k}}}+\left|a_{k+1}\right|^{\frac{1}{\alpha_{k}}}\right)^{2}}\right\}^{\frac{1}{2}},
\end{gathered}
$$

where $\left|\omega_{k}^{(1)}\right|=\left|a_{k}\right|^{\frac{1}{\alpha_{k}}},\left|\omega_{k}^{(2)}\right|=\left|a_{k+1}\right|^{\frac{1}{\alpha_{k}}},\left|\omega_{k}^{(1)}-\omega_{k}^{(2)}\right|=\left|a_{k}\right|^{\frac{1}{\alpha_{k}}}+\left|a_{k+1}\right|^{\frac{1}{\alpha_{k}}}$. Taking into account the fact that

$$
\prod_{k=1}^{n} \frac{1}{2}\left(\left|\frac{a_{k}}{a_{k+1}}\right|^{\frac{1}{2 \alpha_{k}}}+\left|\frac{a_{k+1}}{a_{k}}\right|^{\frac{1}{2 \alpha_{k}}}\right)\left|a_{k}\right|=\prod_{k=1}^{n} \chi\left(\left|\frac{a_{k}}{a_{k+1}}\right|^{\frac{1}{2 \alpha_{k}}}\right)\left|a_{k}\right|=\mathcal{L}\left(A_{n}\right),
$$

we obtain the following inequality

$$
\begin{gathered}
J_{n}(\gamma) \leqslant 2^{n} \cdot\left(\prod_{k=1}^{n} \alpha_{k}\right) \cdot \mathcal{L}\left(A_{n}\right) \times \\
\times \prod_{k=1}^{n}\left\{\left(r\left(\Omega_{k}^{(0)}, 0\right) r\left(\Omega_{k}^{(\infty)}, \infty\right)\right)^{\gamma \alpha_{k}^{2}} \cdot \frac{r\left(\Omega_{k}^{(1)}, \omega_{k}^{(1)}\right) \cdot r\left(\Omega_{k}^{(2)}, \omega_{k}^{(2)}\right)}{\left(\left|a_{k}\right|^{\frac{1}{\alpha_{k}}}+\left|a_{k+1}\right|^{\frac{1}{\alpha_{k}}}\right)^{2}}\right\}^{\frac{1}{2}} .
\end{gathered}
$$

Equality in the last inequality is achieved when equality is realized in the inequalities (4)-(6) for all $k=\overline{1, n}$. Based on the last relation, Theorem 4.1.1 in [1], Corollary 4.1.3 in [1], and the invariance of the functional

$$
\left(\frac{r\left(B_{1}, a_{1}\right) r\left(B_{3}, a_{3}\right)}{\left|a_{1}-a_{3}\right|^{2}}\right)^{\gamma}\left(\frac{r\left(B_{2}, a_{2}\right) r\left(B_{4}, a_{4}\right)}{\left|a_{2}-a_{4}\right|^{2}}\right)
$$


we have

$$
\begin{gathered}
J_{n}(\gamma) \leqslant\left(\frac{2}{\sqrt{\gamma}}\right)^{n} \cdot\left(\prod_{k=1}^{n} \alpha_{k} \sqrt{\gamma}\right) \cdot \mathcal{L}\left(A_{n}\right) \times \\
\times \prod_{k=1}^{n}\left\{\left(r\left(\widetilde{\Omega}_{k}^{(0)}, 0\right) r\left(\widetilde{\Omega}_{k}^{(\infty)}, \infty\right)\right)^{\gamma \alpha_{k}^{2}} \cdot \frac{r\left(\widetilde{\Omega}_{k}^{(1)}, \widetilde{\omega}_{k}^{(1)}\right) \cdot r\left(\widetilde{\Omega}_{k}^{(2)}, \widetilde{\omega}_{k}^{(2)}\right)}{\left(\left|a_{k}\right|^{\frac{1}{\alpha_{k}}}+\left|a_{k+1}\right|^{\frac{1}{\alpha_{k}}}\right)^{2}}\right\}^{\frac{1}{2}},
\end{gathered}
$$

where the domains $\widetilde{\Omega}_{k}^{(0)}, \widetilde{\Omega}_{k}^{(\infty)}, \widetilde{\Omega}_{k}^{(1)}, \widetilde{\Omega}_{k}^{(2)}$ and points $0, \infty, \widetilde{\omega}_{k}^{(1)}, \widetilde{\omega}_{k}^{(2)}$, are, respectively, the circular domains and the poles of the quadratic differential

$$
Q(z) d z^{2}=-\frac{z^{4}+2\left(1-\frac{2}{\gamma \alpha_{k}^{2}}\right) z^{2}+1}{z^{2}\left(z^{2}+1\right)^{2}} d z^{2} .
$$

Each term in the braces of the last inequality is a value of the functional

$$
K_{\tau}=\left[r\left(B_{0}, 0\right) r\left(B_{\infty}, \infty\right)\right]^{\tau^{2}} \cdot \frac{r\left(B_{1}, a_{1}\right) r\left(B_{2}, a_{2}\right)}{\left|a_{1}-a_{2}\right|^{2}}
$$

on the system of nonoverlapping domains $\left\{\widetilde{\Omega}_{k}^{(0)}, \widetilde{\Omega}_{k}^{(1)}, \widetilde{\Omega}_{k}^{(2)}, \widetilde{\Omega}_{k}^{(\infty)}\right\}$, and the corresponding system of points $\left\{0, \widetilde{\omega}_{k}^{(1)}, \widetilde{\omega}_{k}^{(2)}, \infty\right\}(k=\overline{1, n})$.

An estimate of the functional (7) in the case of fixed poles was first obtained in [6], and then in the papers [9,15]. On the basis of Lemma 4.1.2 [1], we get the estimate

$$
K_{\tau} \leqslant \Phi(\tau), \quad \tau \geqslant 0
$$

where $\Phi(\tau)=\tau^{2 \tau^{2}} \cdot|1-\tau|^{-(1-\tau)^{2}} \cdot(1+\tau)^{-(1+\tau)^{2}}$. Then

$$
\begin{gathered}
J_{n}(\gamma) \leqslant\left(\frac{2}{\sqrt{\gamma}}\right)^{n} \cdot\left(\prod_{k=1}^{n} \alpha_{k} \sqrt{\gamma}\right)\left[\prod_{k=1}^{n} \Phi\left(\tau_{k}\right)\right]^{1 / 2}= \\
=\left(\frac{2}{\sqrt{\gamma}}\right)^{n} \cdot\left[\prod_{k=1}^{n}\left(\tau_{k}^{2 \tau_{k}^{2}+2} \cdot\left|1-\tau_{k}\right|^{-\left(1-\tau_{k}\right)^{2}} \cdot\left(1+\tau_{k}\right)^{-\left(1+\tau_{k}\right)^{2}}\right)\right]^{\frac{1}{2}},
\end{gathered}
$$

where $\tau_{k}=\sqrt{\gamma} \cdot \alpha_{k}, k=\overline{1, n}$.

Consider the function $S(x)=x^{2 x^{2}+2} \cdot|1-x|^{-(1-x)^{2}} \cdot(1+x)^{-(1+x)^{2}}$. The function $S(x)$ is logarithmically convex on the interval $\left[0, x_{0}\right], x_{0} \approx 0.88441$. Now we consider an extremal problem

$$
\prod_{k=1}^{n} S\left(x_{k}\right) \longrightarrow \max , \quad \sum_{k=1}^{n} x_{k}=2 \sqrt{\gamma}, \quad x_{k}=\alpha_{k} \sqrt{\gamma}
$$


Let $X^{(0)}=\left\{x_{k}^{(0)}\right\}_{k=1}^{n}$ be an arbitrary extremal point of the problem. The following result holds (obtained similarly [12]):

$$
\Psi^{\prime}\left(x_{1}^{(0)}\right)=\Psi^{\prime}\left(x_{2}^{(0)}\right)=\ldots=\Psi^{\prime}\left(x_{n}^{(0)}\right),
$$

where $\Psi^{\prime}(x)=4 x \ln (x)-2(x-1) \ln |x-1|-2(x+1) \ln (x+1)+\frac{2}{x}($ see Fig. 1).

Further it will be necessary for us to show that the following condition holds:

$$
x_{1}^{(0)}=x_{2}^{(0)}=\cdots=x_{n}^{(0)} \text { for all } \gamma \in\left(0, \gamma_{n}\right] .
$$

Let $\Psi^{\prime}(x)=t, y_{0} \leqslant t<0, y_{0} \approx-1.06$. We find a solution of equation $\Psi^{\prime}(x)=t_{k}, k=\overline{1,53}$. Since $\forall t_{k} \in\left[y_{0}, 0\right)$, it follows that the equation has two solutions $x_{1}(t) \in\left(0, x_{0}\right], x_{2}(t) \in\left(x_{0}, \infty\right]$.

Consider the following values of $t: t_{1}=-0.02, t_{2}=-0.04, t_{3}=-0.06$, $t_{4}=-0.08, \cdots, t_{52}=-1.04, t_{53}=y_{0}$. Direct calculations are presented in Table 1.

Consider the case $n=2$. From the analysis of the tabular data for $n=2$, we get that the minimum of the sum $x_{1}\left(t_{k}\right)+x_{2}\left(t_{k+1}\right)$ is achieved for the interval $[-0.62 ;-0.64]$ and is equal to 1.709336 (see Table 2 ). The relation $x_{1}(t)+x_{2}(t)=2 \sqrt{\gamma}$ holds for each $\gamma \in(0 ; 0.73]$. Let $\gamma=0.73$; then the value $2 \sqrt{\gamma}$ is less than the minimum 1.709336. Thus, for $n=2$ and $\gamma \in(0 ; 0.73]$, we obtain that $x_{2}$ does not belong to $\left(x_{0}, \infty\right)$, that is $x_{1}$ and $x_{2}$ belong to the interval $\left(0, x_{0}\right]$ and $x_{1}=x_{2}$. From inequalities (8) and (9) for $n=2$, we have

$$
J_{2}(\gamma) \leqslant \frac{4}{\gamma} \cdot S\left(\frac{2 \sqrt{\gamma}}{2}\right) .
$$

For $n=3$, the minimum of the value $2 x_{1}\left(t_{k}\right)+x_{2}\left(t_{k+1}\right)$ on the whole graph is achieved on the interval $[-0.48 ;-0.50]$ and is equal to 2.381211 (see Table 3). Similarly, $2 x_{1}(t)+x_{2}(t)=2 \sqrt{\gamma}$. Let $\gamma=1.41$; then $2 \sqrt{\gamma}=2.3748$. Thus, for $\gamma \in(0 ; 1.41]$ the situation $x_{2} \in\left(x_{0}, \infty\right)$ is not possible. In this way, we obtain $x_{1}, x_{2}, x_{3} \in\left(0, x_{0}\right]$ and $x_{1}=x_{2}=x_{3}$.

Then, taking into account the inequalities (8) and (9) for $n=3$, we have

$$
J_{3}(\gamma) \leqslant\left(\frac{2}{\sqrt{\gamma}}\right)^{3}\left[S\left(\frac{2 \sqrt{\gamma}}{3}\right)\right]^{3 / 2} .
$$

Similarly, the situation holds for all $\gamma \in\left(0, \gamma_{n}\right], n=4,5,6$. 
A. K. Bakhtin, I. V. Denega

\begin{tabular}{|c|c|c|c|c|c|c|c|}
\hline$k$ & $t_{k}$ & $x_{1}\left(t_{k}\right)$ & $x_{2}\left(t_{k}\right)$ & $k$ & $t_{k}$ & $x_{1}\left(t_{k}\right)$ & $x_{2}\left(t_{k}\right)$ \\
\hline 0 & 0 & 0.581421 & $\infty$ & 27 & -0.54 & 0.671495 & 1.047944 \\
\hline 1 & -0.02 & 0.584192 & 2.607677 & 28 & -0.56 & 0.675680 & 1.041549 \\
\hline 2 & -0.04 & 0.586996 & 2.095431 & 29 & -0.58 & 0.679954 & 1.035639 \\
\hline 3 & -0.06 & 0.589833 & 1.849825 & 30 & -0.6 & 0.684325 & 1.030184 \\
\hline 4 & -0.08 & 0.592706 & 1.696659 & 31 & -0.62 & 0.688797 & 1.025157 \\
\hline 5 & -0.1 & 0.595614 & 1.588941 & 32 & -0.64 & 0.693377 & 1.020539 \\
\hline 6 & -0.12 & 0.598559 & 1.507710 & 33 & -0.66 & 0.698072 & 1.016313 \\
\hline 7 & -0.14 & 0.601542 & 1.443586 & 34 & -0.68 & 0.702890 & 1.012468 \\
\hline 8 & -0.16 & 0.604564 & 1.391304 & 35 & -0.7 & 0.707842 & 1.008999 \\
\hline 9 & -0.18 & 0.607626 & 1.347643 & 36 & -0.72 & 0.712936 & 1.005911 \\
\hline 10 & -0.2 & 0.610729 & 1.310499 & 37 & -0.74 & 0.718185 & 1.003228 \\
\hline 11 & -0.22 & 0.613876 & 1.278433 & 38 & -0.76 & 0.723604 & 1.001015 \\
\hline 12 & -0.24 & 0.617066 & 1.250421 & 39 & -0.78 & 0.729208 & 0.999457 \\
\hline 13 & -0.26 & 0.620302 & 1.225709 & 40 & -0.8 & 0.735017 & 0.997390 \\
\hline 14 & -0.28 & 0.623585 & 1.203729 & 41 & -0.82 & 0.741053 & 0.994797 \\
\hline 15 & -0.3 & 0.626917 & 1.184045 & 42 & -0.84 & 0.747345 & 0.991762 \\
\hline 16 & -0.32 & 0.630299 & 1.166313 & 43 & -0.86 & 0.753926 & 0.988295 \\
\hline 17 & -0.34 & 0.633734 & 1.150260 & 44 & -0.88 & 0.760838 & 0.984381 \\
\hline 18 & -0.36 & 0.637223 & 1.135664 & 45 & -0.9 & 0.768138 & 0.979982 \\
\hline 19 & -0.38 & 0.640770 & 1.122345 & 46 & -0.92 & 0.775896 & 0.975038 \\
\hline 20 & -0.4 & 0.644375 & 1.110153 & 47 & -0.94 & 0.784212 & 0.969461 \\
\hline 21 & -0.42 & 0.648041 & 1.098962 & 48 & -0.96 & 0.793228 & 0.963114 \\
\hline 22 & -0.44 & 0.651772 & 1.088668 & 49 & -0.98 & 0.803162 & 0.955787 \\
\hline 23 & -0.46 & 0.655569 & 1.079182 & 50 & -1 & 0.814378 & 0.947120 \\
\hline 24 & -0.48 & 0.659437 & 1.070427 & 51 & -1.02 & 0.827585 & 0.936407 \\
\hline 25 & -0.5 & 0.663378 & 1.062338 & 52 & -1.04 & 0.844608 & 0.921828 \\
\hline 26 & -0.52 & 0.667396 & 1.054860 & 53 & -1.06 & 0.884406 & 0.884406 \\
\hline
\end{tabular}

Table 1: Two solutions of the equation $\Psi^{\prime}(x)=t_{k}, k=\overline{1,53}$

From Table 1 , for an arbitrary $n \geqslant 7$, the following inequality holds:

$$
(n-1) x_{1}\left(t_{k}\right)+x_{2}\left(t_{k+1}\right)>n x_{1}\left(t_{k}\right)+\left(x_{2}\left(t_{k+1}\right)-x_{1}\left(t_{k}\right)\right)>0.58 n,
$$

since $x_{1}\left(t_{k}\right) \geqslant 0.5830$ and $x_{2}\left(t_{k+1}\right)-x_{1}\left(t_{k}\right) \geqslant 0$. Using the condition

$$
(n-1) x_{1}(t)+x_{2}(t)=2 \sqrt{\gamma_{n}},
$$

we assume that $2 \sqrt{\gamma_{n}}=0.58 n$. Thus, $\gamma_{n}=0.084 n^{2}$, that is, when $\gamma \in\left(0 ; 0.084 n^{2}\right]$ then the sum $(n-1) x_{1}(t)+x_{2}(t)$ is less than $0.58 n$. Thus, for $n \geqslant 7$ and $\gamma \in\left(0, \gamma_{n}\right]$, we obtain

$$
J_{n}(\gamma) \leqslant\left(\frac{2}{\sqrt{\gamma}}\right)^{n}\left[S\left(\frac{2 \sqrt{\gamma}}{n}\right)\right]^{n / 2}
$$




\begin{tabular}{|c|c|c|c|c|c|}
\hline$k$ & $t_{k}$ & $x_{1}\left(t_{k}\right)+x_{2}\left(t_{k+1}\right)$ & $k$ & $t_{k}$ & $x_{1}\left(t_{k}\right)+x_{2}\left(t_{k+1}\right)$ \\
\hline 0 & 0 & & 27 & $-0,54$ & 1,715340 \\
\hline 1 & $-0,02$ & 3,189098 & 28 & $-0,56$ & 1,713044 \\
\hline 2 & $-0,04$ & 2,679623 & 29 & $-0,58$ & 1,711318 \\
\hline 3 & $-0,06$ & 2,436820 & 30 & $-0,6$ & 1,710138 \\
\hline 4 & $-0,08$ & 2,286492 & 31 & $-0,62$ & 1,709482 \\
\hline 5 & $-0,1$ & 2,181647 & 32 & $-0,64$ & $\mathbf{1 , 7 0 9 3 3 6}$ \\
\hline 6 & $-0,12$ & 2,103324 & 33 & $-0,66$ & 1,709690 \\
\hline 7 & $-0,14$ & 2,042145 & 34 & $-0,68$ & 1,710540 \\
\hline 8 & $-0,16$ & 1,992846 & 35 & $-0,7$ & 1,711889 \\
\hline 9 & $-0,18$ & 1,952207 & 36 & $-0,72$ & 1,713753 \\
\hline 10 & $-0,2$ & 1,918125 & 37 & $-0,74$ & 1,716163 \\
\hline 11 & $-0,22$ & 1,889163 & 38 & $-0,76$ & 1,719200 \\
\hline 12 & $-0,24$ & 1,864297 & 39 & $-0,78$ & 1,723061 \\
\hline 13 & $-0,26$ & 1,842775 & 40 & $-0,8$ & 1,726598 \\
\hline 14 & $-0,28$ & 1,824031 & 41 & $-0,82$ & 1,729814 \\
\hline 15 & $-0,3$ & 1,807630 & 42 & $-0,84$ & 1,732815 \\
\hline 16 & $-0,32$ & 1,793230 & 43 & $-0,86$ & 1,735640 \\
\hline 17 & $-0,34$ & 1,780559 & 44 & $-0,88$ & 1,738307 \\
\hline 18 & $-0,36$ & 1,769398 & 45 & $-0,9$ & 1,740820 \\
\hline 19 & $-0,38$ & 1,759569 & 46 & $-0,92$ & 1,743176 \\
\hline 20 & $-0,4$ & 1,750923 & 47 & $-0,94$ & 1,745356 \\
\hline 21 & $-0,42$ & 1,743337 & 48 & $-0,96$ & 1,747326 \\
\hline 22 & $-0,44$ & 1,736709 & 49 & $-0,98$ & 1,749015 \\
\hline 23 & $-0,46$ & 1,730953 & 50 & -1 & 1,750281 \\
\hline 24 & $-0,48$ & 1,725996 & 51 & $-1,02$ & 1,750785 \\
\hline 25 & $-0,5$ & 1,721775 & 52 & $-1,04$ & 1,749413 \\
\hline 26 & $-0,52$ & 1,718238 & 53 & $-1,06$ & 1,729015 \\
\hline & & & & & \\
\hline
\end{tabular}

Table 2: Minimum of the sum $x_{1}\left(t_{k}\right)+x_{2}\left(t_{k+1}\right), k=\overline{1,53}$

The equality case is straightforward to verify. Theorem 1 is proved.

From Theorem 1, we obtain the following results.

Corollary 1. Let $n \in \mathbb{N}, n \geqslant 2, \gamma \in\left(0, \gamma_{n}\right], \gamma_{2}=0.7304, \gamma_{3}=1.4175$, $\gamma_{4}=2.2983, \gamma_{5}=3.3683, \gamma_{6}=4.6244$, and $\gamma_{n}=0.084 n^{2}, n \geqslant 7$. Then for any $n$-radial system of points $A_{n}=\left\{a_{k}\right\}_{k=1}^{n}$ such that $\mathcal{L}\left(A_{n}\right)=$ $=1$, and any system of mutually non-overlapping domains $B_{0}, B_{\infty}, B_{k}$, $a_{0}=0 \in B_{0} \subset \overline{\mathbb{C}}, \infty \in B_{\infty} \subset \overline{\mathbb{C}}, a_{k} \in B_{k} \subset \overline{\mathbb{C}}, k=\overline{1, n}$, the inequality (2) holds. Equality is attained in the same case as in Theorem 1.

Corollary 2. Under the conditions of Theorem 1, the following inequa- 
lity holds:

$$
\left[r\left(B_{0}, 0\right) r\left(B_{\infty}, \infty\right)\right]^{\gamma} \prod_{k=1}^{n} r\left(B_{k}, a_{k}\right) \leqslant\left(\frac{4}{n}\right)^{n} \frac{\left(\frac{4 \gamma}{n^{2}}\right)^{\frac{2 \gamma}{n}}}{\left|1-\frac{4 \gamma}{n^{2}}\right|^{\frac{2 \gamma}{n}+\frac{n}{2}}}\left|\frac{n-2 \sqrt{\gamma}}{n+2 \sqrt{\gamma}}\right|^{2 \sqrt{\gamma}} .
$$

Equality in this inequality is achieved when $0, \infty, a_{k}$ and $B_{0}, B_{\infty}, B_{k}$, $k=\overline{1, n}$, are, respectively, poles and circular domains of the quadratic differential (3).

\begin{tabular}{|c|c|c|c|c|c|}
\hline$k$ & $t_{k}$ & $2 x_{1}\left(t_{k}\right)+x_{2}\left(t_{k+1}\right)$ & $k$ & $t_{k}$ & $2 x_{1}\left(t_{k}\right)+x_{2}\left(t_{k+1}\right)$ \\
\hline 0 & 0 & & 27 & -0.54 & 2.382735 \\
\hline 1 & -0.02 & 3.770519 & 28 & -0.56 & 2.384539 \\
\hline 2 & -0.04 & 3.263814 & 29 & -0.58 & 2.386998 \\
\hline 3 & -0.06 & 3.023816 & 30 & -0.6 & 2.390093 \\
\hline 4 & -0.08 & 2.876325 & 31 & -0.62 & 2.393807 \\
\hline 5 & -0.1 & 2.774353 & 32 & -0.64 & 2.398133 \\
\hline 6 & -0.12 & 2.698938 & 33 & -0.66 & 2.403067 \\
\hline 7 & -0.14 & 2.640704 & 34 & -0.68 & 2.408612 \\
\hline 8 & -0.16 & 2.594388 & 35 & -0.7 & 2.414780 \\
\hline 9 & -0.18 & 2.556771 & 36 & -0.72 & 2.421594 \\
\hline 10 & -0.2 & 2.525751 & 37 & -0.74 & 2.429099 \\
\hline 11 & -0.22 & 2.499892 & 38 & -0.76 & 2.437386 \\
\hline 12 & -0.24 & 2.478172 & 39 & -0.78 & 2.446665 \\
\hline 13 & -0.26 & 2.459841 & 40 & -0.8 & 2.455806 \\
\hline 14 & -0.28 & 2.444333 & 41 & -0.82 & 2.464831 \\
\hline 15 & -0.3 & 2.431215 & 42 & -0.84 & 2.473869 \\
\hline 16 & -0.32 & 2.420146 & 43 & -0.86 & 2.482985 \\
\hline 17 & -0.34 & 2.410858 & 44 & -0.88 & 2.492232 \\
\hline 18 & -0.36 & 2.403133 & 45 & -0.9 & 2.501659 \\
\hline 19 & -0.38 & 2.396792 & 46 & -0.92 & 2.511314 \\
\hline 20 & -0.4 & 2.391692 & 47 & -0.94 & 2.521252 \\
\hline 21 & -0.42 & 2.387712 & 48 & -0.96 & 2.531538 \\
\hline 22 & -0.44 & 2.384750 & 49 & -0.98 & 2.542243 \\
\hline 23 & -0.46 & 2.382725 & 50 & -1 & 2.553443 \\
\hline 24 & -0.48 & 2.381565 & 51 & -1.02 & 2.565162 \\
\hline 25 & -0.5 & $\mathbf{2 . 3 8 1 2 1 1}$ & 52 & -1.04 & 2.576998 \\
\hline 26 & -0.52 & 2.381615 & 53 & -1.06 & 2.573623 \\
\hline
\end{tabular}

Table 3: Minimum of the sum $2 x_{1}\left(t_{k}\right)+x_{2}\left(t_{k+1}\right), k=\overline{1,53}$

Corollary 3. Let $n \in \mathbb{N}, n \geqslant 2, \gamma \in\left(0, \gamma_{n}\right], \gamma_{2}=0.7304, \gamma_{3}=1.4175$, $\gamma_{4}=2.2983, \gamma_{5}=3.3683, \gamma_{6}=4.6244$, and $\gamma_{n}=0.084 n^{2}, n \geqslant 7$. Then, for any other points of the unit circle $|w|=1$ and any set of mutually 
non-overlapping domains $B_{0}, B_{\infty}, B_{k}, a_{0}=0 \in B_{0} \subset \overline{\mathbb{C}}, \infty \in B_{\infty} \subset \overline{\mathbb{C}}$, $a_{k} \in B_{k} \subset \overline{\mathbb{C}}, k=\overline{1, n}$, the inequality (2) holds. Equality is attained in the same case as in Theorem 1.

If we consider a sufficiently strict restriction on the distribution of the angles $\alpha_{k}, k=\overline{1, n}$, then we can get a stronger result.

Let $y_{0} \approx 0.884414$ be a root of the equation

$$
\ln \frac{y^{2}}{1-y^{2}}=\frac{1}{y^{2}}
$$

Then the following proposition is true.

Theorem 2. Let $n \in \mathbb{N}, n \geqslant 2, \gamma \in\left(0, \gamma_{n}\right), \gamma_{n}=\frac{1}{4} y_{0}^{2} n^{2}$. Then for any $n$-radial system of points $A_{n}=\left\{a_{k}\right\}_{k=1}^{n}$ such that $\mathcal{L}\left(A_{n}\right)=1$, $0<\alpha_{k} \leqslant y_{0} / \sqrt{\gamma}$, where $y_{0}$ is a root of equation (11), $k=\overline{1, n}$, and for any collection of pairwise nonoverlapping domains $B_{0}, B_{\infty}, B_{k}$, $a_{0}=0 \in B_{0} \subset \overline{\mathbb{C}}, \infty \in B_{\infty} \subset \overline{\mathbb{C}}, a_{k} \in B_{k} \subset \overline{\mathbb{C}}, k=\overline{1, n}$, the inequality (10) holds. Equality is attained in the same case as in Corollary 2.

Proof. The proof of Theorem 2 practically repeats the proof of Theorem 1 , only the logarithmic convexity of the function $S(x)$ on the segment $\left(0, y_{0}\right]$ and relation below are used in the final stage of the proof. The reation is

$$
\frac{1}{n} \sum_{k=1}^{n} \ln S\left(x_{k}\right) \leqslant \ln S\left(\frac{\sum_{k=1}^{n} x_{k}}{n}\right)
$$

It is equivalent to

$$
\ln \left(\prod_{k=1}^{n} S\left(x_{k}\right)\right)^{\frac{1}{n}} \leqslant \ln \left(S\left(\frac{2}{n} \sqrt{\gamma}\right)\right)
$$

Equality in this inequality is attained if

$$
\tau_{1}=\tau_{2}=\ldots=\tau_{n}=\frac{2 \sqrt{\gamma}}{n},
$$

i. e., if $\alpha_{k}=\frac{2}{n}, k=\overline{1, n}$. In this case, relation (7) yields

$$
J_{n}(\gamma) \leqslant J_{n}^{0}(\gamma)=\left(\frac{4}{n}\right)^{n}\left[\left(r\left(D_{0}, 0\right) r\left(D_{\infty}, \infty\right)\right)^{\frac{4 \gamma}{n^{2}}} \cdot \frac{r\left(D_{1},-i\right) r\left(D_{2}, i\right)}{|(-i)-i|^{2}}\right]^{\frac{n}{2}}
$$


where $D_{0}, D_{\infty}, D_{1}$ and $D_{2}$ are the circular domains of the quadratic differential

$$
Q(z) d z^{2}=-\frac{\frac{4 \gamma}{n^{2}} z^{4}+2\left(\frac{4 \gamma}{n^{2}}-2\right) z^{2}+\frac{4 \gamma}{n^{2}}}{z^{2}\left(z^{2}+1\right)^{2}} d z^{2}
$$

From whence, we have, eventually,

$$
J_{n}(\gamma) \leqslant\left(\frac{2}{\sqrt{\gamma}}\right)^{n}\left[S\left(\frac{2}{n} \sqrt{\gamma}\right)\right]^{\frac{n}{2}}
$$

Using a specific formula for $S(x)$, we get the basic inequality of Theorem 2 . Changing the variable in (12) by the formula $z=-i w^{\frac{n}{2}}$, we get the quadratic differential (3). The sign of equality in inequality (10) is verified directly. Theorem 2 is proved.

Corollary 1. Let $n \in \mathbb{N}, n \geqslant 2, \gamma \in\left(0, \gamma_{n}\right], \gamma_{n}=0.19 n^{2}$. Then for any $n$-radial system of points $A_{n}=\left\{a_{k}\right\}_{k=1}^{n}$ such that $\mathcal{L}\left(A_{n}\right)=1$, $0<\alpha_{k} \leqslant y_{0} / \sqrt{\gamma}, y_{0} \approx 0.88441, k=\overline{1, n}$, and any set of mutually nonoverlapping domains $B_{0}, B_{\infty}, B_{k}, a_{0}=0 \in B_{0} \subset \overline{\mathbb{C}}, \infty \in B_{\infty} \subset \overline{\mathbb{C}}$, $a_{k} \in B_{k} \subset \overline{\mathbb{C}}, k=\overline{1, n}$, the inequality (2) holds. Equality is attained in the same case as in Theorem 1.

Consider the following problem, which was formulated as an open problem in the case $\gamma=1$ in the paper by Dubinin [7].

Problem 2. Find, for any fixed value of $\gamma \in(0, n]$, the maximum of the functional

$$
r^{\gamma}\left(B_{0}, 0\right) \prod_{k=1}^{n} r\left(B_{k}, a_{k}\right)
$$

where $B_{0}, B_{1}, B_{2}, \ldots, B_{n}, n \geqslant 2$, is any system of pairwise non-overlapping domains in $\overline{\mathbb{C}}$, where the domains $B_{1}, \ldots, B_{n}$ have symmetry with respect to the unit circle, $a_{0}=0,\left|a_{k}\right|=1, k=\overline{1, n}, a_{k} \in B_{k} \subset \overline{\mathbb{C}}, k=\overline{0, n}$; describe all extremals of the functional.

This problem was solved for $\gamma=1$ and $n \geqslant 2$ by Kovalev [13, 14]. The following theorem substantially complements the results of the papers $[4,13,14]$. We obtain the following results assuming that $B_{0} \subset U$ (here $U$ denotes the unit circle).

Theorem 3. Let $n \in \mathbb{N}, n \geqslant 2, \gamma \in\left(0, \gamma_{n}\right), \gamma_{n}=\frac{1}{2} y_{0}^{2} n^{2}$. Then, for any $n$-radial system of points $A_{n}=\left\{a_{k}\right\}_{k=1}^{n}$, such that $\left|a_{k}\right|=1$, 
$0<\alpha_{k} \leqslant y_{0} / \sqrt{\gamma}$, where $y_{0}$ is a root of equation (11), $k=\overline{1, n}$, and any set of mutually non-overlapping domains $B_{0}, B_{k}, a_{0}=0 \in B_{0} \subset U$, $a_{k} \in B_{k} \subset \overline{\mathbb{C}}, k=\overline{1, n}$, where the domains $B_{k}$ have symmetry with respect to the unit circle $|w|=1$ for all $k=\overline{1, n}$, the following inequality holds:

$$
r^{\gamma}\left(B_{0}, 0\right) \prod_{k=1}^{n} r\left(B_{k}, a_{k}\right) \leqslant r^{\gamma}\left(\Lambda_{0}, 0\right) \prod_{k=1}^{n} r\left(\Lambda_{k}, \lambda_{k}\right) .
$$

Equality in (13) is attained when $0, \lambda_{k}$ and $\Lambda_{0}, \Lambda_{k}, k=\overline{1, n}$, are, respectively, the poles and the circular domains of the quadratic differential

$$
Q(w) d w^{2}=-\frac{\gamma w^{2 n}+2\left(n^{2}-\gamma\right) w^{n}+\gamma}{w^{2}\left(w^{n}-1\right)^{2}} d w^{2} .
$$

Proof. Note (see [6, p.59]) that if the domains $B_{k}$ have symmetry with respect to the unit circle $|w|=1$ for all $k=\overline{1, n}$, and the domain $B_{0} \subset$ $U$, then the extremal problem for the functional $r^{\gamma}\left(B_{0}, 0\right) \prod_{k=1}^{n} r\left(B_{k}, a_{k}\right)$ can be reduced, by easy transformations, to the study of the functional $r^{\gamma / 2}\left(B_{0}, 0\right) r^{\gamma / 2}\left(B_{\infty}, \infty\right) \prod_{k=1}^{n} r\left(B_{k}, a_{k}\right)$. Thus, using this property and proofs of Theorem 1 and Theorem 2, we obtain the result of Theorem 3.

Using Corollary 3 and proofs of Theorem 3 and Theorem 1, it is not difficult to obtain the following result.

Theorem 4. Let $n \in \mathbb{N}, \gamma \in\left(0, \gamma_{n}\right], \gamma_{2}=1.4608, \gamma_{3}=2.8350$, $\gamma_{4}=4.5966, \gamma_{5}=6.7366, \gamma_{6}=9.2488, \gamma_{n}=0.168 n^{2}, n \geqslant 7$. Then, for any other points of the unit circle $|w|=1$ and any system of mutually non-overlapping domains $B_{0}, B_{k}, a_{0}=0 \in B_{0} \subset U, a_{k} \in B_{k} \subset \overline{\mathbb{C}}$, $k=\overline{1, n}$, where the domains $B_{k}$ have symmetry with respect to the unit circle $|w|=1$ for all $k=\overline{1, n}$, the following inequality holds:

$$
r^{\gamma}\left(B_{0}, 0\right) \prod_{k=1}^{n} r\left(B_{k}, a_{k}\right) \leqslant\left(\frac{4}{n}\right)^{n} \frac{\left(\frac{2 \gamma}{n^{2}}\right)^{\frac{\gamma}{n}}}{\left|1-\frac{2 \gamma}{n^{2}}\right|^{\frac{n}{2}+\frac{\gamma}{n}}}\left|\frac{n-\sqrt{2 \gamma}}{n+\sqrt{2 \gamma}}\right|^{\sqrt{2 \gamma}} .
$$

Equality in the inequality is achieved when $a_{k}$ and $B_{k}, k=\overline{0, n}$, are, respectively, poles and circular domains of the quadratic differential (14). 


\section{References}

[1] Bakhtin A. K., Bakhtina G. P., Zelinskii Yu. B. Topological-algebraic structures and geometric methods in complex analysis. Zb. prats of the Inst. of Math. of NASU, 2008. (in Russian) DOI: https://doi.org/10.13140/RG. 2.1 .1660 .6242 .

[2] Bakhtin A. K., Denega I. V. Some estimates of the functionals for $n$-radial systems of points. Zb. prats of the Inst. of Math. of NASU, 2011, vol. 8, no. 1, pp. $12-21$. (in Russian)

[3] Bakhtina G. P., Dvorak I. Y., Denega I. V. About the product of inner radii of pairwise non-overlapping domains. Dopov. Nac. akad. nauk Ukr., 2016, no. 1, pp. 7 -11. DOI: https://doi.org/10.15407/dopovidi2016. 01.007.

[4] Bakhtina G. P. On the conformal radii of symmetric nonoverlapping regions. Modern issues of material and complex analysis, K.: Inst. Math. of NAS of Ukraine, 1984, pp. 21-27. (in Russian)

[5] Denega I. V. Some inequalities for inner radii of partially non-overlapping domains. Dopov. Nac. akad. nauk Ukr., 2012, no. 5, pp. 19-22. (in Russian)

[6] Dubinin V. N. Separating transformation of domains and problems on extremal decomposition. Notes scientific. sem. Leningr. Dep. of Math. Inst. AN USSR., 1988, vol. 168, pp. 48-66. (in Russian); translation in J. Soviet Math., 1991, vol. 53, no. 3, pp. 252-263. DOI: https://doi.org/10. $1007 / \mathrm{BF} 01303649$.

[7] Dubinin V. N. Symmetrization method in geometric function theory of complex variables. Successes Mat. Science, 1994, vol. 49, no. 1(295), pp. 3-76. (in Russian); translation in Russian Math. Surveys. 1994, vol. 1, pp. 1-79. DOI: https://doi.org/10.1070/RM1994v049n01ABEH002002.

[8] Dubinin V. N. Condenser capacities and symmetrization in geometric function theory. Birkhäuser/Springer, Basel, 2014. DOI: : https://doi.org/ 10.1007/978-3-0348-0843-9.

[9] Emelyanov E. G. On the Problem of Maximizing the Product of Powers of Conformal Radii Nonoverlapping Domains. J. Math. Sci. (N.Y.), 2004, vol. 122, no. 6, pp. 3641-3647. DOI: https://doi.org/10.1023/B: JOTH. 0000035239.55516 .08$.

[10] Goluzin G. M. Geometric theory of functions of a complex variable. Amer. Math. Soc. Providence, R.I., 1969.

[11] Jenkins J. Univalent functions and conformal mapping. Moscow:Publishing House of Foreign Literature, 256, 1962. (in Russian) DOI: : https://doi . org/10.1007/978-3-642-88563-1. 
[12] Kovalev L. V. On the problem of extremal decomposition with free poles on a circle. Dal'nevost. Mat. Zh., 1996, no. 2, pp. 96-98. (in Russian)

[13] Kovalev L. V. On the inner radii of symmetric nonoverlapping domains. Izv. Vyssh. Uchebn. Zaved. Mat., 2000, no. 6, pp. 77-78. (in Russian)

[14] Kovalev L. V. On three nonoverlapping domains. Dal'nevost. Mat. Zh., 2000, no. 1, pp. 3-7. (in Russian)

[15] Kuzmina G. V. Problems on extremal decomposition of the riemann sphere. Notes scientific. Sem. Leningr. Dep. of Math. Inst. AN USSR., 2001, vol. 276, pp. 253-275. (in Russian); translation in J. Math. Sci. (N.Y.), 2003, vol. 118, no. 1, pp. 4880 -4894. DOI: https://doi.org/10.1023/A: 1025580802209.

[16] Lavrent'ev M. A. On the theory of conformal mappings. Tr. Sci. Inst An USSR, 1934, vol. 5, pp. 159-245. (in Russian)

Received September 19, 2018.

In revised form, September 21, 2018.

Accepted December 28, 2018.

Published online January 11, 2019.

Institute of Mathematics of the National Academy of Sciences of Ukraine Department of complex analysis and potential theory 01004 Ukraine, Kiev-4, 3, Tereschenkivska st.

A. K. Bakhtin

E-mail: abahtin@imath.kiev.ua

I. V. Denega

E-mail: iradenega@gmail.com 